\title{
A rare cause of foot pain: Osteoid osteoma of the cuboid bone
}

\section{Nadir Bir Ayak Ăgrısı Sebebi: Kuboid Kemikte Osteoid Osteoma}

\author{
Orhan Balta ${ }^{1}$, Murat Aş̧̧ı ${ }^{1}$, Mete Gedikbaş ${ }^{1}$, Bora Bostan ${ }^{1}$ \\ ${ }^{1}$ Gaziosmanpaşa Üniversitesi Tıp Fakültesi, Ortopedi Ve Travmatoloji A.D, Tokat
}

Dergiye Ulaşma Tarihi: 201.12.2016 Dergiye Kabul Tarihi:23.01.2017 Doi: 10.5505/aot.2017.74436

\section{ÖZET}

Osteoid osteoma, sık görülen benign karakterli primer kemik tümörüdür. Hastalar sıklıkla ağrı şikâyeti ile başvururlar. Literatürde vücudun çeşitli kemiklerinde bildirilmiş olgu sunumları mevcuttur. Ekstremite ağrısı yakınması olan hastaların ayırıcı tanısında osteoid osteomayı aklımızın bir köşesinde tutmak faydalı olacaktır. Osteoid osteomadan şüphelendiğimizde yapılacak ince kesit BT tanı konulmasında yararlıdır. Literatürde 3 adet kuboid kemik tutulumu görülen osteoid osteoma vakasına ulaşabildik. Bu yazıda, geçmeyen ayak ağrısı nedeniyle başvuran 26 yaşında hastada kuboid kemikte saptanan osteoid osteoma vakasını sunmaktayız.

Anahtar Kelimeler: Osteoid osteoma, cuboid kemik, ağrı

\begin{abstract}
Osteoid osteoma is a common benign primary bone tumor. Patients frequently present with pain. There are cases reported in various bones in the literature. It is useful to keep in mind the osteoid osteoma in the differential diagnosis of patients with limb pain. The thin section computed tomography will be usefull when we suspect osteoid osteoma. In the literature we have reached three cases of osteoid osteoma related with cuboid bone. We present a case of osteoid osteoma in a 26-year-old patient in cuboid bone due to unusual foot pain.
\end{abstract}

Keywords: Osteoid osteoma, cuboid bone, limb pain

\section{Giriş}

Osteoid osteoma ilk kez 1935 yilında Jaffe ve ark. tarafından tanımlanmıştır (1). Osteoid osteoma primer kemik tümörlerinin \%34'ünden sorumlu olup, tüm benign kemik tümörlerinin \%10-14'ünü meydana getirirler (2). Osteoid osteoma s1klıkla alt ekstremitede ve uzun kemiklerde görülür. Osteoid osteoma olgularının \%50-60'1 femur ve tibia lokalizasyonludur (3). Uzun kemiklerden özellikle proksimal femur ve tibiayı sıklıkla tutar. Tarsal kemikler içerisinde en sık tutulan kemik talus olup \%2-10 oranında tutulum görülmektedir (2). Yaşamın ikinci ve üçüncü dekadlarında daha sık oranda görülmektedir. Erkeklerde kadınlara oranla daha sik görülmekte ve yaklaşık erkek:kadın oranı 2:1'dir(2). Elde ve ayakta nadiren görülmektedir. Literatürde osteoid osteomanın teşhis ve tedavisinde ortalama 36 aya kadar gecikmeler bildirilmiştir. Görüntüleme tekniklerindeki gelişmelere paralel olarak kemik taraması ile semptomların ortaya çıkmasından tanıya kadar geçen süreyi 35 aydan 12 aya kadar önemli ölçüde azalmıştır (4). Literatür incelendiğinde kuboid kemik tutulumu olan üç adet vaka bildirilmiştir.

$\mathrm{Bu}$ yazıda kliniğimize sağ ayak bileğinde ağrı şikâyeti ile başvuran kuboid kemik tutulumu olan osteoid osteoma olgusu sunulmaktadır.

\section{Olgu}

26 yaşında erkek hasta sağ ayak bileği antero lateralinde ağrı ve yürümede zorluk şikayetleriyle kliniğimize başvurdu. 4 yıl önce ayak bileği burkulması sonrasında başlayan hafif ağrısının son 2 yıldır sürekli hale geldiği ve ağrı kesici ilaçlar kullanmak zorunda kaldığını belirtmekteydi. Analjezik kullanımı olmaksızın yürüyemediğini ifade eden hasta daha önce birçok kez doktora gittiğini belirtmekteydi. Fizik muayenesinde belirgin antaljik yürüyüşü olan hastanın ayak bileği hareket açıklığının tam olduğu gözlendi. Ayak lateralinde palpasyonla ağrı dışında fizik 
muayene bulgusu yoktu. 6 ay önce çektirmiş olduğu ayak MR tetkikinde bir patoloji gözlenmedi. Ancak yapılan tetkikin kalın kesit olduğu ve ağrılı bölgeye spesifik olmadığı fark edildi. Bunun üzerine tekrar çekilen ince kesitli ayak MR tetkikinde kuboid kemik süperomedial kesiminde heterojen kemik iliği ödemi ve subkortikal kitle tespit edildi.

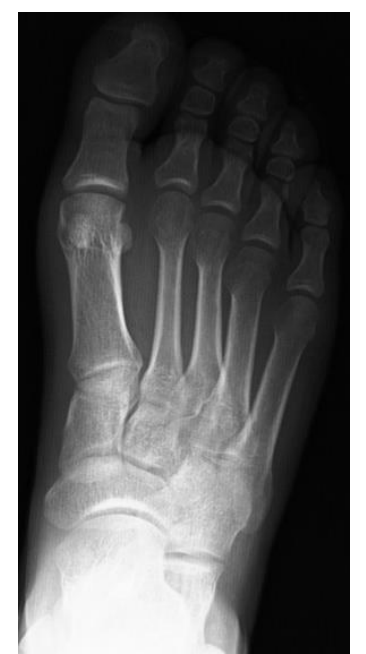

Şekil 1: Ameliyat öncesi çekilen AP ayak grafisinde patolojik görünüm izlenmemekte.

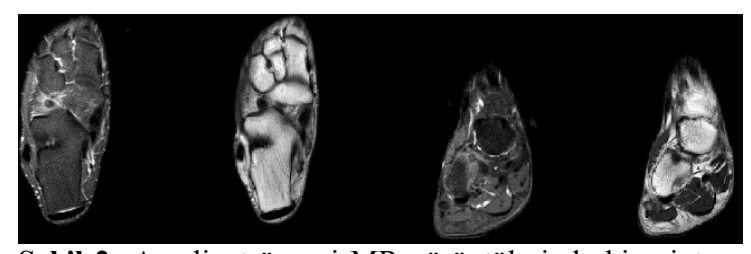

Şekil 2: Ameliyat öncesi MR görüntülerinde hiperintens sinyal değişimleri ve nidus görülmekte.

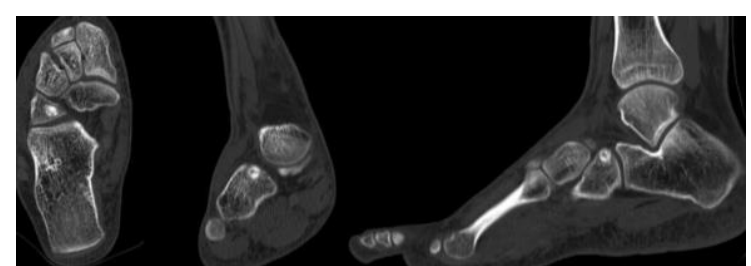

Şekil 3: Ameliyat öncesi BT kesitlerinde karakteristik nidus görünümü mevcut.

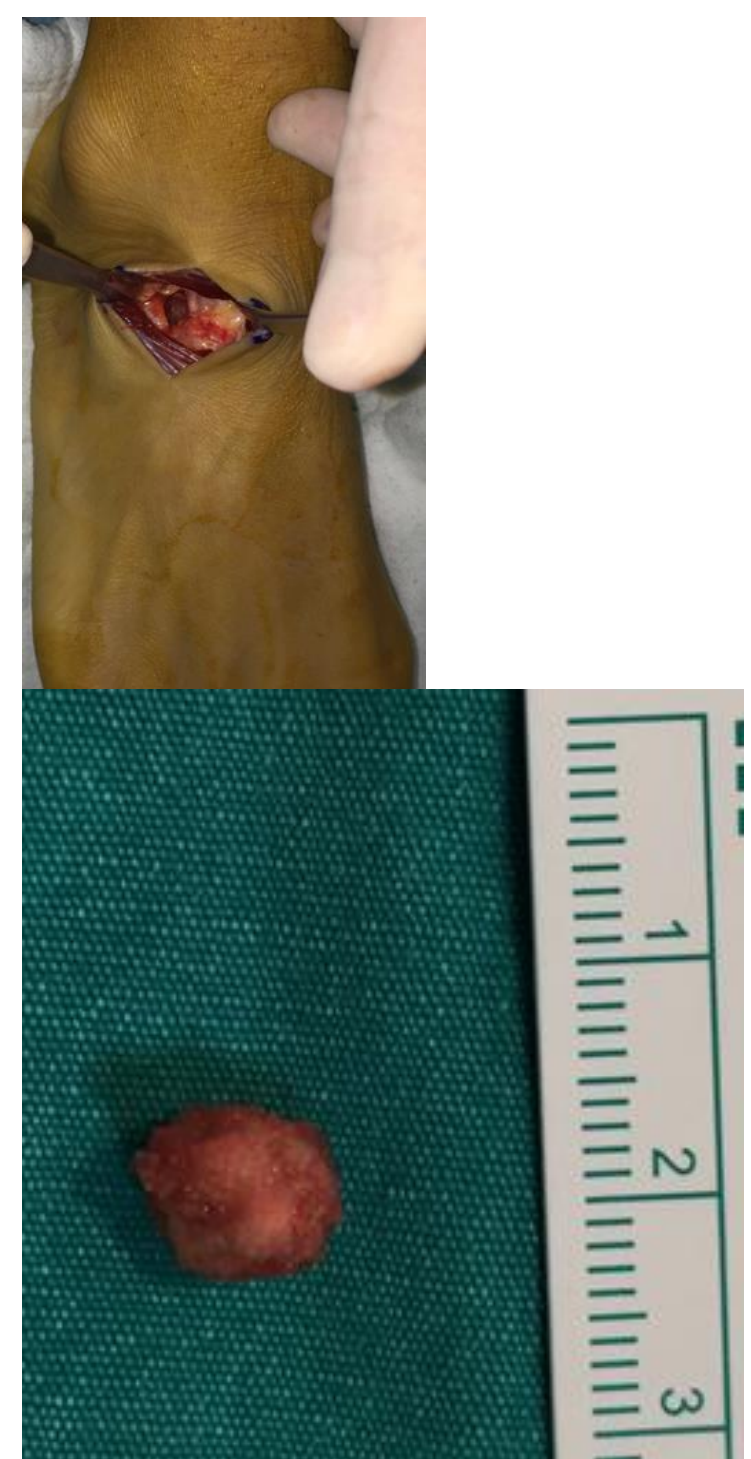

Şekil 4: Ameliyat esnasındaki görüntülerinde karakteristik nidus görünümü mevcut

Rejyonel anestezi altında ayak dorsolateralinden longitudinal insizyonla girilerek kuboid kemikteki kitle en-blok eksize edildi. Operasyon sonrasında çekilen kontrol MR ve radyografi incelemelerinde kitlenin tamamen eksize edildiği görüldü. Alınan biyopsi materyallerinin patolojik incelemesinde sonuç ön tanımızı destekler nitelikte osteoid osteoma olduğu belirtildi.

Ameliyat sonrasında kısa dönemde hastanın şikayetlerinde dramatik azalma görüldü. Ameliyat sonras1 1. y1l kontrolünde hastanın şikayetinin olmadığı ve radyolojik tetkiklerde rekürrens gelişmediği görüldü. 


\section{Tartışma}

Osteoid osteoma primer kemik tümörlerinin \%3-4'ünden sorumlu olup, tüm benign kemik tümörlerinin \%10-14'ünü meydana getirirler. Özellikle proksimal femur ve tibia olmak üzere siklıkla uzun kemikleri tutar. Osteoid osteoma olgularının çoğunluğu uzun kemik korteksinde görülmesine rağmen, her kemikte olabilir. Tek bir kemik veya birkaç kemik içerebilir. Osteoid osteomalar genellikle 1.5-2 cm'den küçüktür, ancak osteoid açısından zengin bir nidus ile karakterizedir. Nidus sklerotik normal kemik ile sınırlandırılmıştır (5-7).

Alt ekstremitede kalça en s1k tutulan bölgedir. Özellikle de femur inter trokanterik veya intra kapsüler bölgeler vakaların üçte ikisinde tutulur $(8,9)$. Osteoid osteoma nadirde olsa el, elbileği, falankslar, ayak kemiklerini tutabilir (10-12). Ayak tutulumun nadir olmas1 ve ayak kemikleri içerisinde en sık tutulumun talusta görülmesi tanı konulmasında gecikmeye yol açmaktadır. Yapılan sistematik bir derlemede ortalama tanı alma süresinin 22 ay olduğu ve bu sürenin 1-120 ay arasında değiştiği bildirilmiştir $(2,13)$.

Osteoid osteoma her yaşta görülmekle birlikte genellikle 10-30 yaşlarındaki kişilerde daha sik olarak görülmektedir. Barei ve arkadaşları yaptıkları çalışmada osteoidosteomalarının \%70'inin 20 yaşından küçük hastalarda ortaya çıktığını belirtmiştir (3). 30 yaşın üzerindeki bireyler osteoidosteoma vakalarının \%13'ünü oluştururken, 5 yaşından küçük çocuklar vakaların yaklaşık \%3'ünü oluşturur $(14,15)$.

Dikkatli bir öykü, detaylı fizik muayene ve görüntüleme ile tanı desteklenmelidir (16). Hastalar özellikle gece artan ve non-steroid anti enflamatuar ilaç kullanımı ile azalan ağrıdan yakınmaktadırlar (17). Osteoid osteomanın en yaygin semptomu salisilat ve non-steroid anti enflamatuar tedaviye iyi yanıt veren nokturnal ağrılardır. Lokal hassasiyet, yumuşak dokuda şişlik, sıcaklık artışı, dinlenme anında ağrı ilk başvuru anında görülebilecek bulgulardır (18). Osteoid osteoma ağrının yanı sıra çok çeşitli klinik şekillerde prezente olabilir. Fiz hattına yakın lezyonlar anormal büyümeye neden olabilir. İntra kapsüler yerleşimli lezyonlar osteo artrite neden olabilir. Vertebral yerleşimli lezyonlar skolyoza neden olabilir. Bu yüzden osteoid osteoma akılda tutulması gereken bir tanıdır. Kirchner ve ark. salisilatlara verdikleri yanıtın \%30-75 arasında değişebileceğini bildirmektedirler (19). Carpintero-Benitez ve ark. non-steroidlere kiyasla, siklooksijenaz2(COX-2) inhibitörlerine ağrının iyi yanıt verdiğini belirtmişlerdir (20). Saville ve ark. aspirin, indometasin, ibuprofen ve fenoprofen ile denemelerden sonra naproksenin terapötik dozlarına iyi yanıt verdiğini bildirmektedirler (21).

Görüntülemede tipik olarak periost reaksiyonu ve kortikal kalınlaşma görülmektedir. MR genellikle tanıy abartabilir. BT osteoid osteomanın kesin teşhisi için birincil tanı aracıdır (22). Sans ve arkadaşları, BT'nin vakaların \%74'ünde osteoid osteoma teşhisini doğrulamada yardımeı olduğunu bildirmiştir (23). Szendroi ve ark. eklem içi lezyonların tanısında yaklaşık \%66, eklem dışı lezyonlarda $\% 90$ doğruluk bildirmiştir (24).

Osteoid osteoma kortikal, kansellöz ve subperiosteal olarak 3 tipte siniflandırılır. Uzun kemiklerde bulunan osteoid osteomalarin trakortikal olup yüksek miktarda subperiosteal reaksiyon oluştururlar. Buna karşın ayakta görülen osteoid osteomalar sıklıkla kansellöz veya subperiosteal olup periosteal reaksiyon minimal olabilir veya görülmemektedir (18). Osteoid osteomanın patognomonik BT bulgusu fokal, santral yerleşimli kalsifikasyon gösteren nidus ve perinidal skleroz görülmesidir. Nidusun merkezinde yüksek konsantrasyonda prostoglandin üretimi mevcut olup artmış gerilim, sinir uçlarında ağrıya neden olan ödem oluşumuna neden olmaktadır. Bu durum osteoid osteomanın non-steroid anti enflamatuar tedaviye olan iyi yanıtını açıklamaktadır (25).

Literatürde bulunan Gürkan ve ark. (26), Aydoğan ve ark. (27) ile Chakraverty ve ark. (28) tarafindan takdim edilen üç vaka sunumunda kuboid kemik yerleşimli osteoid osteoma vakalarında hastalar bizim hastamızda olduğu gibi ikinci ve üçüncü dekad içerisinde olan hastalardı ve ayak bileği burkulması sonrasında şikayetleri ortaya çıkan hastalardı. Hastalar tıpkı bizim vakamızda olduğu gibi osteoid osteomanın atipik yerleşimli olmasından dolayı geç tanı almıştı. Chakraverty ve ark. tarafindan sunulan vakada bizim hastamızda olduğu gibi kemik iliği ödemi mevcuttu ve çekilen radyografilerde herhangi bir patolojik görüntü yoktu. Tanı sonrası cerrahi tedavi ile hastaların şikayetleri tamamen 
gerilemiş ve 1 y1llık takiplerinde rekürrens izlenmemiştir.

Sonuç olarak osteoid osteoma ayakta çok sık görülmese de uzun süreli ağrısı olan özellikle genç erkek hastalarda ayırıcı tanıda aklımıza gelmelidir. Hastamızda görüldüğü gibi kansellöz tipte olan osteoid osteoma vakalarında direk grafilerde bulgu olmayabilirken uygun çekilmiş BT ve MRI incelemeleri tanı ve tedavi için faydalı olabilir.

\section{Kaynaklar}

1. Jaffe, Henry L. "Osteoid-osteoma: a benign osteoblastic tumor composed of osteoid and atypical bone." Archives of Surgery 31.5 (1935): 709-728.

2. Jordan, Robert W., et al. "Osteoid osteoma of the foot and ankle: A systematic review." Foot and Ankle Surgery 21.4 (2015): 228-234.

3. Barei DP, Moreau G, Scarborough MT, Neel MD. Percutaneous radio frequency thermal ablation of osteoid osteoma. Operative Tech Orthop. 1999 Apr. 9(2):72-8

4. Pettine KA, Klassen RA. Osteoid-osteoma and osteoblastoma of the spine. J Bone Joint Surg Am. 1986 Mar. 68(3):354-61.

5. Gonzalez G, Abril JC, Mediero IG, Epeldegui T. Osteoid osteoma with a multi centric nidus. Int Orthop. 1996. 20(1):61-3.

6. Greenspan A. Benign bone-forming lesions: osteoma, osteoid osteoma, and osteoblastoma. Clinical, imaging, pathologic, and differential considerations. Skeletal Radiol. 1993 Oct. 22(7):485-500.

7. Iceton J, Rang M. An osteoid osteoma in an open distal femoral epiphysis. A case report. Clin Orthop Relat Res. 1986 May. 162-5.

8. Lenke LG, Sutherland CJ, Gilula LA. Osteoid osteoma of the proximal femur: CT-guided preoperative e localization. Orthopedics. 1994 Mar. 17(3):289-92.

9. Ebrahimzadeh MH, Ahmadzadeh-Chabock H, Ebrahimzadeh AR. Osteoid osteoma: a diagnosis for radicular pain of extremities. Orthopedics. 2009 Nov. 32(11):821.

10. Bilgin SS, Yildiz Y, Guclu B, Saglik Y. Osteoid osteoma in the hand: an evaluation of eight patients. Acta Orthop Traumatol Turc. 2004. 38 (3):206-11.

11. Kalb K, Schlor U, Meier M, et al. Osteoid osteoma of the hand and wrist [in German]. Handchir Mikrochir Plast Chir. 2004 Dec. 36(6):405-10.

12. Nanni M, Pantalone O, Zappala A, et al. Diagnostic approach to a rare case of osteoid osteoma of the astragalus. Rays. 2004 Apr-Jun. 29 (2):217-21.

13. Chakraverty, Julian, Namir Al-Mokhtar, and Steven L. James. "Osteoid osteoma of the cuboid managed by percutaneous radio frequency ablation." The Journal of Foot and Ankle Surgery 53.2 (2014): 212-215.

14. Frassica FJ, Waltrip RL, Sponseller PD, et al. Clinicopathologic features and treatment of osteoid osteoma and osteoblastoma in children and adolescents. Orthop Clin North Am. 1996 Jul. 27(3):559-74.

15. Donkol RH, Al-Nammi A, Moghazi K. Efficacy of percutaneous radiofrequency ablation of osteoid osteoma in children. Pediatr Radiol. 2008 Feb. 38(2):180-5.

16. Catani, Fabio, et al. "Osteoid osteoma of the lateral cuneiform bone." Foot \&ankle international 15.10 (1994): 570-572.

17. Houdek, M. T., et al. "Osteoid osteomas of the foot and ankle: a study of patients over a 20-year period." American journal of orthopedics (Belle Mead, NJ) 43.12 (2014): 552-556.

18. Bostan, Bora, et al. "Osteoid osteoma of the trapezium: case report." The Journal of hand surgery 35.4 (2010): 636-638.

19. Kirchner B, Hillmann A, Lottes G, et al. Intraoperative, probe-guided curettage of osteoid osteoma. Eur J Nucl Med. 1993 Jul. 20(7):609-13.

20. Carpintero-Benitez P, Aguirre MA, Serrano MA, Lluch M. Effect of rofecoxib on pain caused by osteoid osteoma. Orthopaedics. 2004 Nov. 27 (11):1188-91.

21. Saville PD. A medical option for the treatment of osteoid osteoma. Arthritis Rheum. 1980 Dec. 23(12):1409-11

22. Athwal GS, Pichora DR, Ellis RE, Rudan JF. A computer-assisted guidance technique for the localization and excision of osteoid osteoma. Orthopedics. 2004 Feb. 27(2):195-7.

23. Sans N, Galy-Fourcade D, Assoun J, et al. Osteoid osteoma: CT-guided percutaneous resection and follow-up in 38 patients. Radiology. 1999 Sep. 212(3):687-92.

24. Szendroi M, Killo K, Antal I, et al. Intra articular osteoid osteoma: clinical features, imaging results, and comparison with extra articular localization. J Rheumatol. 2004 May. 31 (5):957-64.

25. Hosalkar, Harish S., et al. "The diagnostic accuracy of MRI versus CT imaging for osteoid osteoma in children." Clinical orthopaedics and related research 433 (2005): 171-177.

26. Gurkan, Volkan, et al. "Osteoid osteoma of the cuboid bone: a rare cause of foot pain." Acta Orthop Traumatol Turc 45.1 (2011): 66-69.

27. Aydogan, Umur, and Samuel G. Dellenbaugh. "An Unusual Case of Chronic Lateral Foot Pain Following Ankle Inversion Injury Osteoid Osteoma of the Tarsal Cuboid Bone." Foot \& ankle specialist (2014): 1938640013514266.

28. Chakraverty, Julian, Namir Al-Mokhtar, and Steven L. James. "Osteoid osteoma of the cuboid managed by percutaneous radio frequency ablation." The Journal of Foot and Ankle Surgery 53.2 (2014): 212-215. 